\title{
Differences in shaft strain patterns during golf drives due to stiffness and swing effects
}

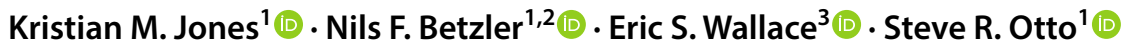

Published online: 5 July 2019

(c) The Author(s) 2019

\begin{abstract}
There is much debate around the role of shaft stiffness in the dynamic response of the club shaft during the golf swing. This study used a novel complex analysis to investigate within- and between-golfer differences in shaft strain patterns for three shaft stiffnesses. Twelve right-handed male golfers, with a handicap less than or equal to five, hit six shots with three driver clubs which differed only in shaft stiffness. Clubs were instrumented to record the shaft strain in the lead/lag and toe/heel directions. The analysis combined these perpendicular components into a single complex function, which enabled the differences between two swings to be characterised by a scale and a rotation component. Within-golfer strain patterns were found to be significantly more consistent than between-golfer, $p<0.01$. Whilst some golfers displayed more similar patterns than others, there were no clear groups of golfers with similar patterns of shaft strain. Between the clubs, shaft strain patterns differed in the scale component, $p<0.01$, rather than the rotation, $p=0.07$.
\end{abstract}

\section{Introduction}

The role of shaft bending during the golf swing has been subject to much debate, with claims that it may facilitate loading and unloading of the shaft like a spring [1], and counterclaims that 'centrifugal stiffening' [2] and the damping provided by the hands [3] would prevent this from occurring. Shaft bending stiffness has been the focus of scientific investigation [4]; however, despite universal acceptance that the club shaft bends during the backswing and the downswing with a recoil as the clubhead approaches impact with the ball, the role of varying shaft stiffness on golfing performance is not yet fully understood [5].

Studies investigating the effects of shaft stiffness on performance include theoretical models [6, 7] and experimental

This article is a part of Topical Collection in Sports Engineering on Measuring Behavior in Sport and Exercise, edited by Dr. Tom Allen, Dr. Robyn Grant, Dr. Stefan Mohr and Dr. Jonathan Shepherd.

Kristian M. Jones

kristianjones@randa.org

Research and Testing, The R\&A, St. Andrews, Fife, UK

2 Qualisys AB, Gothenburg, Sweden

3 Sport and Exercise Sciences Research Institute, Ulster University, Newtownabbey, UK studies, utilising either high-speed photogrammetry [8] or, more commonly, the use of strain gauges attached to the shaft [1, 7, 9-17]. The results of the theoretical studies lack experimental validation whilst overall results are often conflicting [5]; however, it has been shown that shafts of varying flex have distinctly different bending profiles throughout the swing $[8,16,18,19]$. Recent work has suggested that a more flexible shaft makes a greater contribution to clubhead speed than a stiffer shaft but may not result in greater clubhead speed due to reduced angular velocity at the grip [20].

In addition to shaft flex effects on bending properties, it has been noted that there can be marked differences in shaft deflection patterns when comparing swings of different golfers [13]. Various shaft strain profiles, characterised as "single peak", "double peak" and "ramp like", have been suggested to occur during the swing [1], yet these patterns appear to be mainly based on qualitative observations rather than quantifiable results. Jorgensen [2] performed simulations of golf swings that included flexible shafts and found that a small torque supporting the wrist 'uncocking' process, or wrist release, could explain the way that the shaft bends during the downswing. Both Suzuki et al. [21] and Nesbit [22] claim that highly skilled golfers can use joint torque profiles that influence shaft deflection in a way that allows an efficient utilisation of energy stored in the shaft. Koike et al. [23] measured a golfer's torque and force inputs to the shaft directly using an instrumented grip. They only presented 
preliminary results for one scratch golfer, but the trend for the wrist torque to drop towards a zero or negative torque within the last $0.05 \mathrm{~s}$ of the downswing can also be observed in their results.

Apart from a study by our group [9] that provides aggregate strain profiles for a group of golfers for two shaft flexes, no previous studies have been identified that quantitatively define strain profiles for shafts of different flex including within- and between-golfer effects. The purpose of the present study, therefore, was to add to the limited understanding of both golfer and shaft flex effects on shaft strain during the golf swing with a driver club using three shafts of different stiffness. Using a novel complex analysis, the aims of the study were to (1) determine within-golfer strain consistency levels, (2) to compare strain patterns across golfers, and (3) to provide a characterisation of golfers' shaft strain patterns for the three shaft flexes.

\section{Methods}

\subsection{Clubs and testing equipment}

Three driver clubs were assembled with the intention of matching their first moment, termed swing weight; their overall mass; their performance metrics; and their aesthetics, within reasonable ranges. Small changes in club swing weight are generally not perceivable by golfers and have little effect on playing performance [10]. The three shafts featured in this study were advertised as having equal mass but different stiffness. The shaft frequency, rigidity and bend point of several unassembled shafts, of the same design, were measured, and the shafts which matched most closely were chosen for the investigation. The shaft rigidity was measured at a cantilever length of $1 \mathrm{~m}$ and the shaft bend point measured relative to the tip end of the shaft when unassembled. The only characteristic which was designed to vary was the shaft rigidity (often termed shaft flex). The properties are given for the shafts, and the fully assembled club including the strain gauges, associated wiring and markers (Table 1).
The clubheads used for this study were the same generic model and were confirmed to conform to the Rules of Golf [24]. To account for differences between clubheads caused by manufacturing tolerances, the properties of several clubheads of the same model were measured, and those which matched most closely were chosen (Table 2).

Each shaft was equipped with four uniaxial foil strain gauges (Kyowa, Japan), forming two half-bridges. The strain gauges were $2 \mathrm{~mm}$ long, their resistance was $120 \Omega$ and they were placed at the location where the highest magnitude of strain was expected during the swing. This location was the point of maximum bending curvature, as determined during a static shaft bending test, approximately $0.5 \mathrm{~m}$ from the tip end of the shafts, regardless of their stiffness rating (Table 1). The strain gauges were aligned with the longitudinal axis of the shafts and placed so that one pair of strain gauges recorded the lead/lag strain of the shaft and the other pair the toe-up/down strain. A cable connected to the butt end of the club transmitted the strain signals to two P-3500 analogue strain amplifiers (Vishay, USA). Three reflective markers were fixed to the club just below the grip to track the movement of the club throughout the swing (Fig. 1). Strain was recorded synchronously with the club movement via a Qualisys A/D board (Qualisys AB, Sweden) at a sample rate of $960 \mathrm{~Hz}$. Movement trajectories of the reflective markers were recorded using an eight camera ProReflex motion capture system (Qualisys AB, Sweden), operating at $240 \mathrm{~Hz}$. The system was calibrated according to the manufacturer's instructions prior to testing.

Table 2 Clubhead characteristics for the three drivers used for the study. Testing tolerances were $\pm 0.2 \mathrm{~g}, \pm 0.8^{\circ}, \pm 0.8^{\circ}$ and $\pm 50 \mathrm{~g} \mathrm{~cm}^{2}$, respectively

\begin{tabular}{lllll}
\hline Clubhead & Mass $(\mathrm{g})$ & $\operatorname{Loft}\left({ }^{\circ}\right)$ & $\operatorname{Lie}\left({ }^{\circ}\right)$ & MOI $_{\mathrm{z}}\left(\mathrm{g} \mathrm{cm}^{2}\right)$ \\
\hline$' l '$ & 197.0 & 10.8 & 59.5 & 3675 \\
$' r$ & 197.4 & 10.9 & 59.0 & 3687 \\
$' x$ & 198.4 & 11.5 & 58.8 & 3719 \\
\hline
\end{tabular}

Table 1 Club characteristics for the three drivers used for the study. Test tolerances were $\pm 0.2 \mathrm{~g}, \pm 0.5 \mathrm{Nm}^{2}, \pm 1 \mathrm{cpm}, \pm 0.02$ in., \pm 0.1 and $\pm 0.2 \mathrm{~g}$, respectively

\begin{tabular}{|c|c|c|c|c|c|c|c|}
\hline Label & Shaft flex & Shaft mass (g) & $\begin{array}{l}\text { Shaft rigidity } \\
\left(\mathrm{Nm}^{2}\right)\end{array}$ & $\begin{array}{l}\text { Shaft frequency } \\
(\mathrm{cpm})\end{array}$ & Shaft length (m/in.) & Swing weight & Total mass (g) \\
\hline ' $l$ ' & Ladies & 56.8 & 38 & 217 & $1.14 / 45.0$ & C9.7 & 306.8 \\
\hline ' $r$ ' & Regular & 57.2 & 48 & 244 & $1.14 / 45.0$ & C9.6 & 305.6 \\
\hline ' $x$ ' & Extra stiff & 57.7 & 58 & 271 & $1.14 / 45.0$ & D0.0 & 307.7 \\
\hline
\end{tabular}




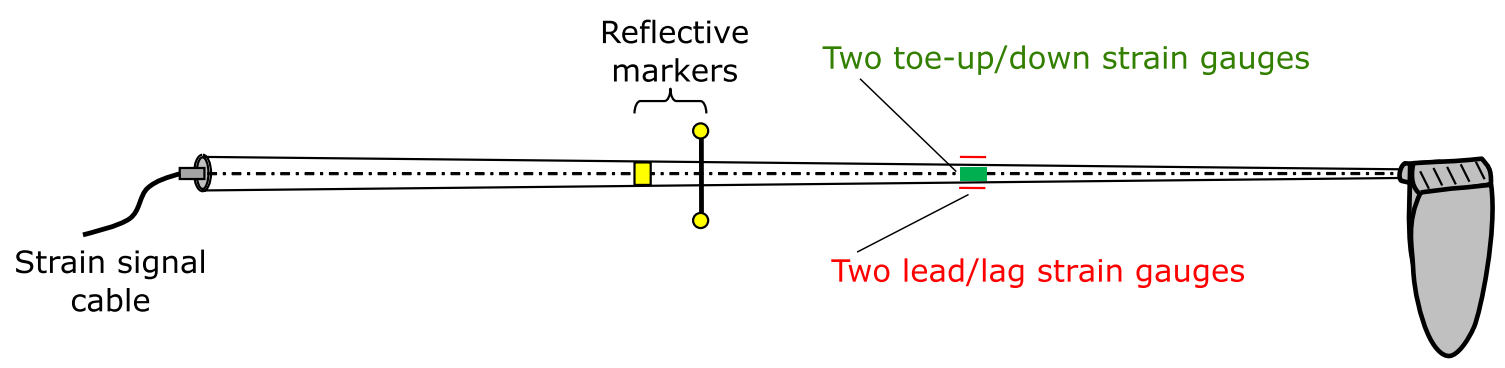

Fig. 1 Schematic of instrumented and assembled golf club

\subsection{Golfer testing}

Twelve right-handed, male golfers, with a handicap less than or equal to five, participated in this study (handicap: $2 \pm 1.8$; age: $34 \pm 10$ years; height: $1.8 \pm 0.1 \mathrm{~m}$ : body mass: $81 \pm 10 \mathrm{~kg}$; all values are mean \pm standard deviation). Professional golfers were assumed to have a handicap of zero. The study was approved by the ethical review board at Edinburgh Napier University, and informed consent was obtained from each golfer prior to testing. After performing their own warm-up routine, golfers performed a set of six swings with each of the three test clubs in a randomised order, determined using the Latin square method [25]. After the three sets, the golfers performed a final set of six swings with the first club used in the main analysis to assess the potential impact of order and fatigue. During each set, each golfer performed a self-selected number of familiarisation swings prior to data recording and repeated the swing if data were not recorded for any reason. The maximum number of swings performed in a set to achieve six valid measurements was nine. Golfers were not made aware of the variable being considered in the study during the test.

\subsection{Data processing}

For each swing, the takeaway event was defined as the moment at which the velocity of the lower shaft marker exceeded $0.2 \mathrm{~m} \mathrm{~s}^{-1}$ and the impact event defined as the sample when the strain rate of lead/lag strain exceeded $-3000 \mathrm{~s}^{-1}$. The strain time series for this period of interest were extracted and the time series aligned at impact. To create time series of equal lengths, the time series were trimmed to the length of the shortest signal from takeaway to impact. The maximum amount of signal trimmed at the start of the time series was $0.3348 \mathrm{~s}$ (331 frames) which equated to $26 \%$ of the total time for that swing. This method was preferred over time normalisation methods, such as resampling to 1000 points, because it did not distort the derivatives of the signal, but this choice did not significantly impact the results of the study. Alternative periods of interest, such as from the top of the back swing, defined as the point at which the clubhead is stationary, or the zero crossing of toe/heel strain component, which generally occurs just prior to the top of the back swing, were also considered and did not significantly impact the results.

\subsection{Complex analysis}

The analysis considered the comparison of strain data between two swings, before extending this analysis to a range of swings. For the $j$ th swing, the toe/heel and lead lag strain were combined into a complex function, such that:

$S^{(j)}(t)=S_{\text {toe }}^{(j)}(t)+i S_{\text {lead }}^{(j)}(t)$

The use of complex analysis is not necessary, but it enabled both components of strain to be considered jointly using simple analytics. Equivalent analysis could be performed in a Cartesian or polar system, but the use of complex analysis facilitated a procedure based on simple arithmetic functions, conveniently simplifying the analysis. To compare the $j$ th swing and the $k$ th swing, the latter was expressed as a linear multiple of the former plus an offset:

$S^{(k)}=A_{j, k} S^{(j)}+B_{j, k}$

where $A_{j, k}, B_{j, k} \in \mathbb{C}$. The constant $A_{j, k}$ can be expressed as

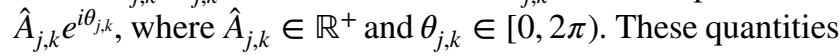
are a scaling of the strain between swings and a rotation of the strain, respectively. The shift, $B_{j, k}$, facilitated the fitting process and in practice it was found that $\left|B_{j, k}\right|=o\left(\hat{A}_{j, k}\right)$. Furthermore, the correlation between $\left|S^{(j)}\right|$ and $\left|S^{(k)}\right|$ was calculated; $C_{j, k}=\operatorname{corr}\left(\left|S^{(j)}\right|,\left|S^{(k)}\right|\right)$. Thus, there were three quantities which could be used to compare two swings: $A_{j, k}$, the scaling, $\theta_{j, k}$, the rotation, and $C_{j, k}$, the correlation. For practical purposes, the scaling is effectively the difference in magnitude between the two swings and the rotation is effectively the amount of lead/lag strain compared to the amount of toe-up/down strain. The correlation indicated the similarity of the two swings.

Comparisons between participants or conditions were performed by calculating all possible pairwise comparisons between the relevant sets; the scaling, rotation and correlation, characterised by the mean, $\mu$, and standard deviation, 
$\sigma$, of these matrices of comparisons. To indicate the comparisons being considered, the following general notation was used: $\left(M_{s}^{i, j}\right)_{x, y}$, with $M$ being the metric being considered (the scaling, rotation or correlation), $s$ being the summary statistic used (either the mean or standard deviation), $i$ and $j$ indicating the players compared (either specifically or generally), and $x$ and $y$ the shafts being compared (either specifically or generally). For example, $\left(\hat{A}_{\mu}^{1,2}\right)_{l, r}$ denoted the mean of the 36 pairwise comparisons of scaling between shots from Golfer 1 using the l-flex shaft and Golfer 2 using the r-flex shaft.

\subsection{Data analysis}

The first aim of the investigation was to determine the within-golfer strain consistency, which was achieved by calculating $\left(C_{\mu}^{k, k}\right)_{z, z}$ and $\left(C_{\sigma}^{k, k}\right)_{z, z}$ for each participant; the mean $(\mu)$ and standard deviation $(\sigma)$ of the correlations for each participant $(k, k)$ with the same shaft $(z, z)$. The second aim of the investigation was to examine the between-golfer strain consistency, which was achieved by comparing $\left(C_{\mu}^{k, k}\right)_{z, z}$ and $\left(C_{\mu}^{k, j}\right)_{z, z}$, with $k \neq j$; the mean correlations for each participant with the same shaft compared to the mean correlations between the participants with the same shaft. The statistical significance of this difference was assessed using a Student's $t$ test. Finally, a characterisation of golfers shaft strain patterns with the different shafts was achieved by comparing $\left(\hat{A}_{\mu}^{k, k}\right)_{z, y}$ and $\left(\theta_{\mu}^{k, k}\right)_{z, y}$ for each shaft combination (' $l$ '- ' $x$ ', ' $r$ '-' $x$ ', ' $l$ '-' $r$ ') to $\left(\hat{A}_{\mu}^{k, k}\right)_{z, z}$ and $\left(\theta_{\mu}^{k, k}\right)_{z, z}$; the average shift and rotation for multiple swings with the same shaft. The statistical significance of differences was assessed using a one-way repeated measures ANOVA, with post hoc Bonferroni adjusted $t$ test. All data analysis and hypothesis testing were performed in MATLAB 2018a (Mathworks, USA). The significance level was set at $p=0.05$ for all statistical tests.

\section{Results}

\subsection{Within-golfer shaft strain}

In repeated shots with the same shaft, all golfers displayed generally similar and consistent strain patterns (Fig. 2); $\left(C_{\mu}^{k, k}\right)_{z, z}=0.983 \pm 0.012$, and $\left(C_{\sigma}^{k, k}\right)_{z, z}=0.013 \pm 0.009$. This was the case for all three shafts studied; $\left(C_{\mu}^{k, k}\right)_{l, l}=0.984$ $\pm 0.012,\left(C_{\sigma}^{k, k}\right)_{l, l}=0.012 \pm 0.011,\left(C_{\mu}^{k, k}\right)_{r, r}=0.981 \pm 0.012$, $\left(C_{\sigma}^{k, k}\right)_{r, r}=0.015 \pm 0.009,\left(C_{\mu}^{k, k}\right)_{x, x}=0.984 \pm 0.011$, and $\left(C_{\sigma}^{k, k}\right)_{x, x}=0.013 \pm 0.009$. Correlations between shots in the
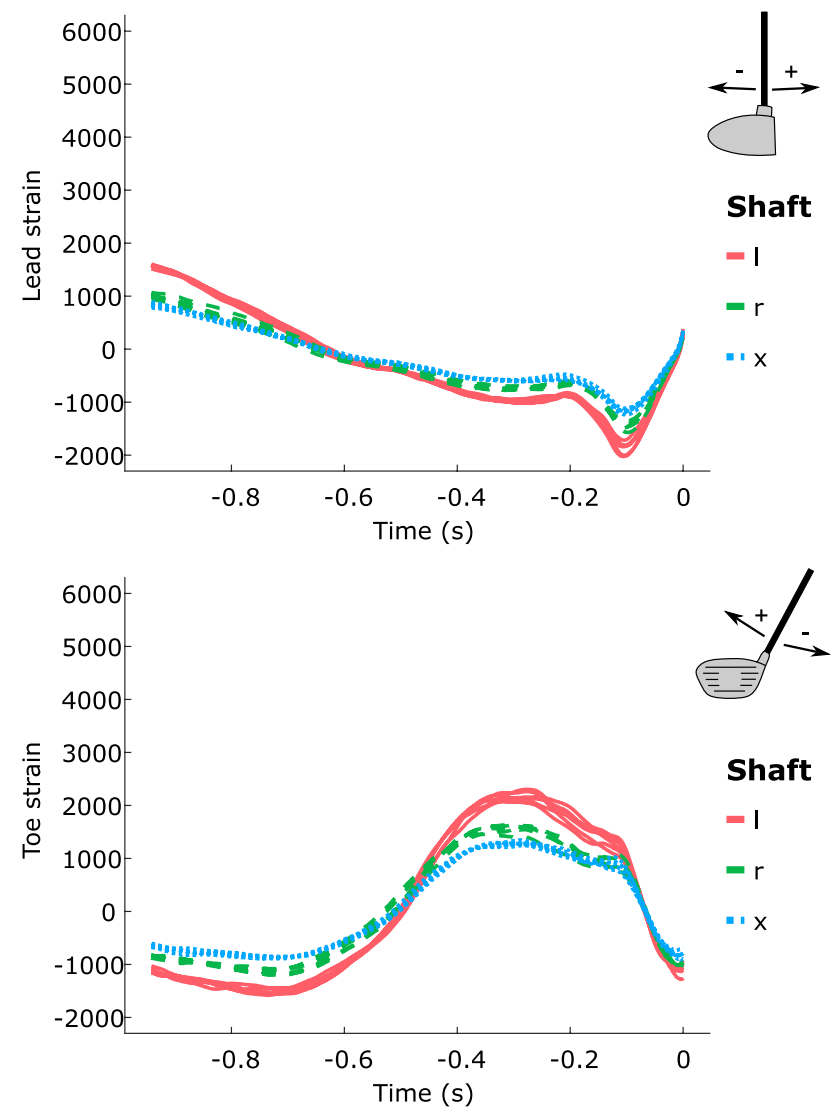

Fig. 2 Lead and toe strain for the three different shafts for Golfer 1. All six swings with each club are displayed. The consistency of within-club patterns of strain displayed by this golfer are typical of those displayed by the cohort

first and last sets, which were completed with the same club, were similar to comparisons between shots in the same set; $\left(C_{\mu}^{k, k}\right)_{z 1, z 2}=0.970 \pm 0.026, t(58)=1.24, p=0.22$.

\subsection{Between-golfer shaft strain}

Shaft strain patterns were significantly less consistent between golfers than within golfers; $t(430)=-12.52$, $p<0.01,\left(C_{\mu}^{k, k}\right)_{z, z}=0.983 \pm 0.012,\left(C_{\mu}^{k, j}\right)_{z, z}=0.489 \pm 0.237$. The variability of the correlations was also significantly higher when comparing between golfers compared to within golfers; $t(430)=11.83, p<0.01,\left(C_{\sigma}^{k, k}\right)_{z, z}=0.013 \pm 0.009$, $\left(C_{\sigma}^{k, j}\right)_{z, z}=0.059 \pm 0.023$. However, whilst all golfers displayed higher correlations with their own shaft loading patterns, there were participants who showed a degree of similarity with each other. For example, Golfer 1 and Golfer 10 showed generally high correlation, $\left(C_{\mu}^{1,10}\right)_{z, z}=0.858 \pm 0.042$, whilst Golfer 4 and Golfer 10 showed much lower correlation, $\left(C_{\mu}^{4,10}\right)_{z, z}=0.142 \pm 0.010$. The corresponding shaft 

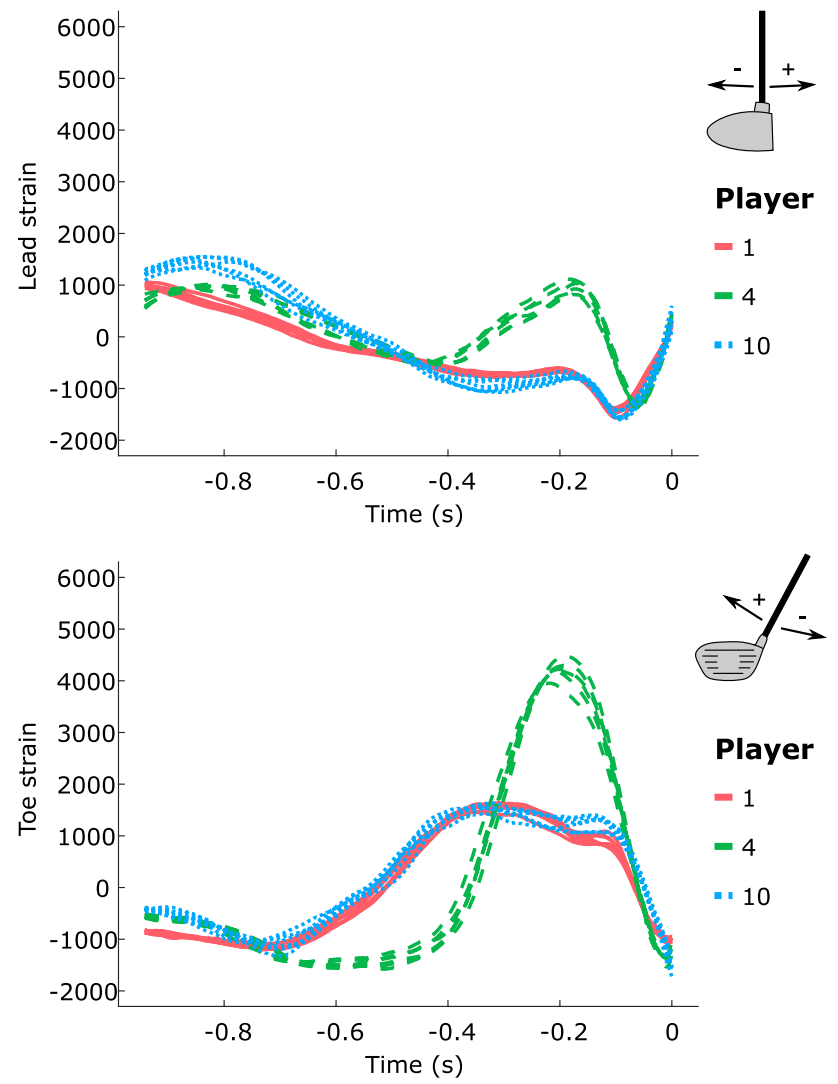

Fig. 3 Lead and toe strain using the r-flex driver for Golfers 1, 4 and 10; all six swings by each golfer are displayed. Lead and toe-up strain are indicated by a positive strain, while lag and toe-down strain are indicated by a negative strain

loading patterns are shown in Fig. 3. The maximum between-golfer correlation was $0.898 \pm 0.006$, between participants 6 and 11, which was lower than the average withingolfer correlation. The similarity of golfers was visualised using a figure in which golfers were positioned based on their average correlation, with golfers who displayed similar patterns located near to one another. Whilst there were similarities between certain participants, the correlations did not separate the participants into obvious groups (Fig. 4).

\subsection{Shaft strain patterns}

Comparing swings with two different shafts, there was generally a good fit when comparing swings from a single individual, shown by high correlations between the compared swings; $\left(C_{\mu}^{k, k}\right)_{z, y}>0.964$. Differences in strain patterns were characterised by a rotation and a change in scaling factor, a reduction in scale and a positive rotation when comparing a less stiff shaft to a stiffer shaft and an opposite increase in scale and negative rotation when comparing a stiffer shaft to a less stiff shaft (Table 3 ). The scale factor, $\left(\hat{A}_{\mu}^{k, k}\right)_{z, y}$ was

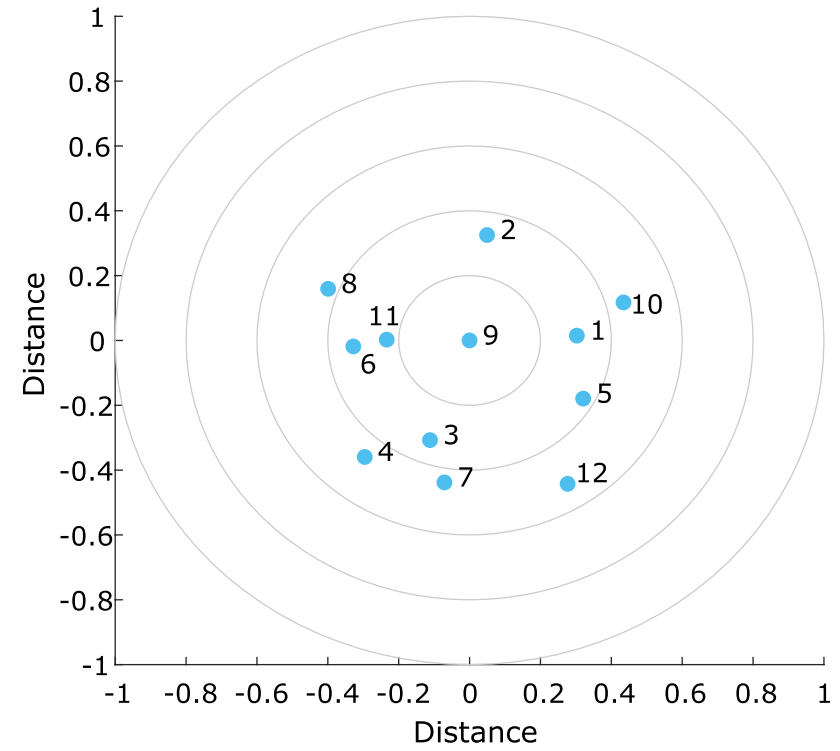

Fig. 4 Similarity of strain patterns between golfers visualised in a two-dimensional space where highly correlated golfers are placed in a similar region. The distance between golfers is approximately $1-\left(C_{\mu}^{k, j}\right)_{z, z}$ and greater distance between golfers indicates lower similarity. Concentric circles are equally spaced to make it easier to judge the distance between golfers. Full details of the method used to construct this figure are included in the "Appendix"

significantly different between comparisons with different shaft stiffness and comparisons with the same shaft stiffness; $F(5,50)=967.70, p<0.01$. Post hoc tests indicated that all comparisons were statistically significant; $p<0.01$. Whilst there was a trend toward a positive rotation when comparing shafts with increasing stiffness, the standard deviation of these differences was much higher than the differences themselves and the differences were not statistically significant; $F(5,50)=2.19, p=0.07$. Thus, the primary difference in shaft strain profile between different shaft stiffnesses was in magnitude; shafts with greater flexibility were associated with a higher magnitude of strain during the golf swing (Fig. 5).

\section{Discussion}

Optimising shaft stiffness on an individual basis may facilitate increased performance [1], but whilst it has been previously shown that shafts of varying stiffness respond differently through the swing $[16,18,19]$, the general consensus is that shaft stiffness does not have a systematic effect on clubhead speed $[5,9,20]$. The main finding of this investigation was that differences in shaft strain during the swing were primarily in the magnitude of the strain. The overall 'pattern' of the strain profile and the ratio of the lead/lag and toe-up/down strain were generally consistent between 
Table 3 Average scaling and rotation factors comparing swings with different flex shafts

\begin{tabular}{|c|c|c|c|c|}
\hline Comparison & $\left(\hat{A}_{\mu}^{k, k}\right)_{z, y}$ & $\left(\hat{A}_{\sigma}^{k, k}\right)_{z, y}$ & $\left(\theta_{\mu}^{k, k}\right)_{z, y}$ & $\left(\theta_{\sigma}^{k, k}\right)_{z, y}$ \\
\hline$' l '-{ }^{\prime} x '$ & $0.601 \pm 0.011$ & $0.015 \pm 0.006$ & $0.717 \pm 1.347$ & $1.870 \pm 0.503$ \\
\hline 'l'-'r' & $0.743 \pm 0.015$ & $0.020 \pm 0.007$ & $0.547 \pm 1.460$ & $1.780 \pm 0.349$ \\
\hline ' $r$ '-‘ $x$ ' & $0.806 \pm 0.016$ & $0.022 \pm 0.007$ & $0.186 \pm 1.872$ & $1.880 \pm 0.580$ \\
\hline
\end{tabular}
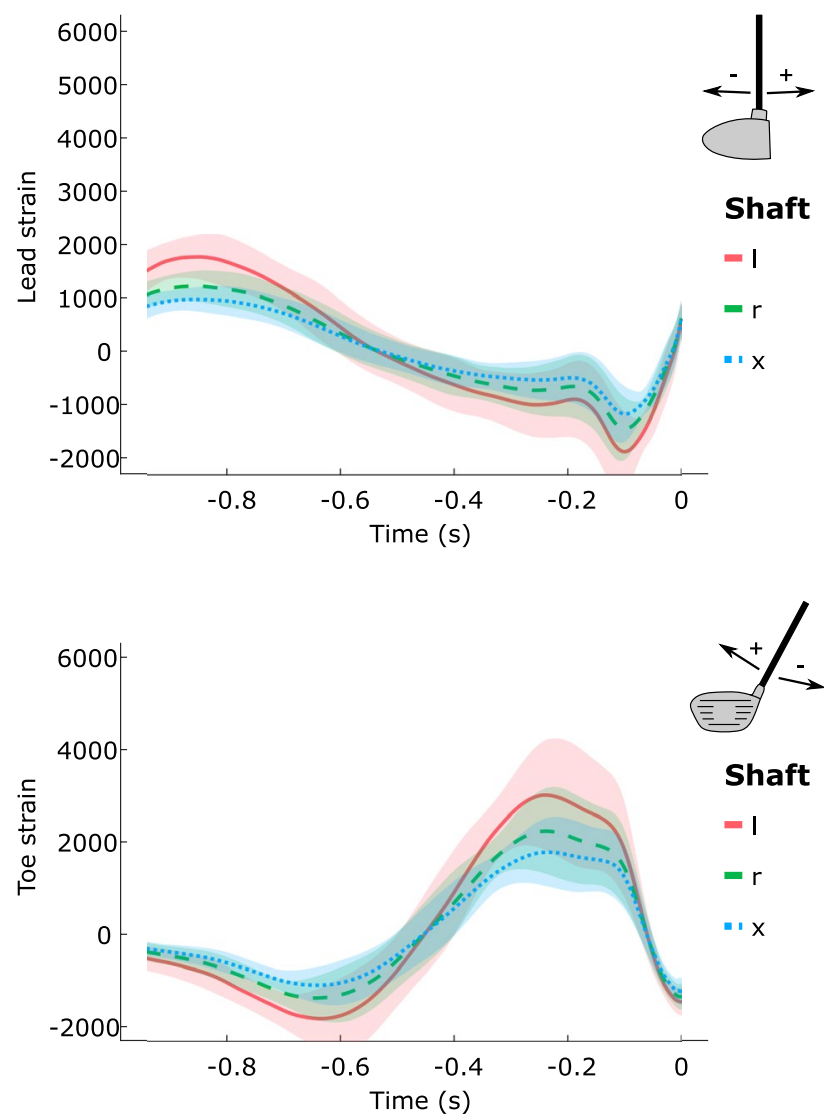

Fig. 5 Average strain patterns for all participants with the three different shafts (mean \pm standard deviation)

clubs for individual golfers. This insight was achieved using a novel complex analysis which considered the lead/lag and toe-up/down components of shaft strain jointly. Whilst separating the two components of strain makes intuitive sense, due to each component's different effect on clubhead presentation at impact, understanding the overall shaft bending dynamics is important.

The maintenance of the shaft strain profile during the swing, found in this investigation, is compatible with results presented by MacKenzie and Boucher [20] which provide an explanation as to the lack of a systematic effect of shaft stiffness on clubhead speed. Whilst the speed of shaft recoil was greater with a more compliant shaft, a result previously reported in the literature [6], this was offset by a decrease in angular velocity at the grip end of the club. That differences were predominantly in the magnitude of the strain, with the strain profile otherwise remaining relatively consistent, supports the suggestion that optimum shaft stiffness may be individually specific [20]. Apart from a change in magnitude, the shaft strain profiles for individual golfers were also highly similar with different stiffness shafts, indicated by high average correlations between the conditions. However, it is important to consider that the kinematics of the swing may be different between the clubs. That is, the golfers' movement patterns may have been altered with the different stiffness shafts, either due to the golfers adapting their swing or due to differences in reaction forces applied by the shaft. Differences in kinematics might explain the reported decrease in angular velocity at the grip end of the club [20] and the discrepancies with results from computer simulation studies [6]. Whilst golfers are typically blinded to the shaft stiffness in human studies, like in this investigation, it is probable that golfers adjust their movements based on the subjective feel of the club. The characteristics of these adjustments are unclear, as this was not the topic of this investigation, but future research could consider these potential differences.

The within-golfer consistency of shaft strain profile was greater than between golfers, consistent with research into movement variability [26]. Indeed, a golfer's shaft strain profile showed greater average similarity to their own swings with a different flex shaft, a minimum correlation of 0.964 , than to any other golfer with the same flex shaft, a maximum correlation of 0.898 . This suggests that the golfers had swings which were reasonably robust to changes in shaft stiffness and other variables, such as the first moment of inertia, swing weight, or the golfers' subjective feel of the club, might be more important considerations in club fitting. However, the effect of changes in shaft stiffness on outcome measures was not considered in this investigation and only golfers in a narrow range of skill were studied.

Butler and Winfield [1] proposed three categories of strain profiles during the swing: single peak, double peak and ramp like. Inspection of individual shaft strain profiles (Fig. 3, for example) showed that characteristics of these patterns were evident in the golfers studied, although classification was sometimes difficult as certain golfers displayed features from multiple categories. Furthermore, whilst the number of golfers studied in this investigation is a limitation, it is worth noting that the correlations between swings 
suggested that the golfers did not fit into three discrete groups. There were no distinct groupings of golfers based on shaft strain correlations, rather golfers' shaft strain profiles were somewhat individual. Larger samples would be required to fully investigate the scientific merit of categorisation in this manner, but the results highlight the individuality of golfers and the need to consider this when working with individual golfers.

\section{Conclusion}

This investigation used a novel method of analysis which facilitated the joint consideration of both the lead/lag and toe-up/down components of shaft strain during the golf swing. The use of complex analysis simplified this analysis and allowed differences between two shaft strain profiles to be characterised by two values, the scale and the rotation. Similarly, the consistency of two shaft strain profiles could be characterised by their correlation. Three different stiffness shafts were considered, but compared to the effect of changing golfer, the effect of a change in shaft stiffness was small and primarily manifested in a difference in the magnitude of shaft strain during the swing, rather than a rotation of the shaft strain or a change in the shaft strain profile. This information may be useful for the provision of custom-fit clubs to golfers. The approach used in this investigation, utilising complex analysis, may also be applied to other sporting examples where the variable of interest is a two-dimensional signal varying over time.

\section{Compliance with ethical standards}

Conflict of interest The authors declare that they have no conflict of interest.

Open Access This article is distributed under the terms of the Creative Commons Attribution 4.0 International License (http://creativeco mmons.org/licenses/by/4.0/), which permits unrestricted use, distribution, and reproduction in any medium, provided you give appropriate credit to the original author(s) and the source, provide a link to the Creative Commons license, and indicate if changes were made.

\section{Appendix: method used to construct Fig. 4}

Figure 4 plotted the similarity of golfer's average strain patterns on a map which located highly similar golfers in similar regions of the plot. The distance between golfers then indicated the average similarity of the golfer's shaft strain patterns. The diagram was constructed to provide an intuitive understanding of how similar shaft strain patterns were and used the following method:
- The correlation, $C_{j, k}=\operatorname{corr}\left(\left|S^{(j)}\right|,\left|S^{(k)}\right|\right)$, was a measure of the similarity between two shaft strain signals.

- As the correlation was calculated between all swings, a matrix which compares the average similarity between each pair of golfers, $\left(C_{\mu}^{k, j}\right)_{z, z}$, could be constructed. This matrix ranged from zero, not similar at all, to one, completely similar.

- Because of intra-individual variability in strain pattern, $\left(C_{\mu}^{k, k}\right)_{z, z}$, the correlation between two swings by the same golfer, was not perfect and the diagonal of this matrix was not equal to one. However, for the purpose of this diagram, the diagonal was set equal to one as a simplification.

- By subtracting one and multiplying by minus one this matrix was converted so that it ranged from zero, completely similar, to one, not similar at all. This matrix was then considered to describe the 'distance' between the golfers, with golfers with a similar shaft strain patterns being 'closer' to each other than golfers with highly different shaft strain patterns.

- Two-dimensional coordinates were resolved from this matrix using multidimensional scaling ( stress $=0.029)$ [27].

- These coordinates were then mapped onto a two-dimensional space, where golfers with a high average correlation are located near to one another and golfers with a low average correlation are far apart (Fig. 4).

\section{References}

1. Butler JH, Winfield DC (1994) The dynamic performance of the golf shaft during the downswing. In: Farrally MR, Cochran AJ (eds) Science and Golf II. E \& FN Spon, New York, pp 259-264

2. Jørgenson T (1970) On the dynamics of the swing of a golf club. Am J Phys 38:644-651

3. Wicks AL, Knight CE, Braunwart P, Neighbors J (1999) The dynamics of a golf club. In: Proceedings of the 17th International Modal Analysis Conference, 3727, p 503

4. Penner AR (2003) The physics of golf. Rep Prog Phys 66:131. https://doi.org/10.1088/0034-4885/66/2/202

5. Worobets J, Stefanyshyn D (2012) The influence of golf club shaft stiffness on clubhead kinematics at ball impact. Sports Biomech 11:239-248. https://doi.org/10.1080/14763141.2012.674154

6. MacKenzie SJ, Sprigings EJ (2009) Understanding the role of shaft stiffness in the golf swing. Sports Eng 12:13-19. https://doi. org/10.1007/s12283-009-0028-1

7. Milne RD, Davis JP (1992) The role of the shaft in the golf swing. J Biomech 25:975-983

8. Mather JSB, Smith MJ, Jowett S et al (2000) Application of a photogrammetric technique to golf club evaluation. Sports Eng 3:37-47. https://doi.org/10.1046/j.1460-2687.2000.00036.x 
9. Betzler NF, Monk SA, Wallace ES, Otto SR (2012) Effects of golf shaft stiffness on strain, clubhead presentation and wrist kinematics. Sports Biomech 11:223-238

10. Harper TE, Roberts JR, Jones R (2005) Driver swingweighting: a worthwhile process? Proc Inst Mech Eng Part B J Eng Manuf 219:385-393. https://doi.org/10.1243/095440505X32247

11. Horwood GP (1994) Golf shafts-a technical perspective. In: Farrally MR, Cochran AJ (eds) Science and golf II. E \& FN Spon, New York, pp 247-258

12. Kojima A, Horii H (1995) Effect of torsional properties of CFRP golf club shafts on the speed and the directional stability of the ball. In: Nakata E, Sakatani Y (eds) Maekawa Z. SAMPE, Yokohama, pp 1328-1334

13. Lee N, Erickson M, Cherveny P (2002) Measurement of the behaviour of a golf club during the golf swing. In: Thain $\mathrm{E}$ (ed) Science and golf IV. Routledge, Abingdon, pp 554-561

14. Newman S, Clay S, Strickland P (1997) The dynamic flexing of a golf club shaft during a typical swing. In: Fourth annual conference on mechatronics and machine vision in practice. pp 265-270

15. Ozawa T, Namiki H, Horikawa N (2002) A study on clubface direction during the golf swing. In: Ujihashi $\mathrm{S}$, Haake $\mathrm{S}$ (eds) The engineering of sport 4. Blackwell Publishing, Oxford, pp 688-694

16. Tsujiuchi N, Koizumi T, Tomii Y (2002) Analysis of the influence of golf club design on the golf swing. In: Ujihashi S, Haake $S$ (eds) The engineering of sport 4. Blackwell Publishing, Oxford, pp 537-544

17. Tsunoda M, Bours RCH, Hasegawa H (2004) Three-dimensional motion analysis and inverse dynamic modelling of the human golf swing. SAE International, Warrendale

18. Masuda M, Kojima S (1994) Kick back effect of club-head at impact. In: Farrally MR, Cochran AJ (eds) Science and golf II. E \& FN Spon, New York, pp 284-289
19. Mather JSB, Jowett S (2000) Three dimensional shape of the golf club during the swing. In: Subic A, Haake S (eds) The engineering of sport-research development and innovation. Blackwell Publishing, London, pp 77-85

20. MacKenzie SJ, Boucher DE (2017) The influence of golf shaft stiffness on grip and clubhead kinematics. J Sports Sci 35:105111. https://doi.org/10.1080/02640414.2016.115726221

21. Suzuki S, Haake SJ, Heller BW (2006) Multiple modulation torque planning for a new golf-swing robot with a skilful wrist turn. Sports Eng 9:201-208. https://doi.org/10.1007/BF02866058

22. Nesbit SM (2005) A three dimensional kinematic and kinetic study of the golf swing. J Sports Sci Med 4:499-519

23. Koike S, Iida H, Shiraki H, Ae M (2006) An instrumented grip handle for golf clubs to measure forces and moments exerted by each hand during swing motion. In: Moritz EF, Haake S (eds) The engineering of sport 6. Springer, New York, pp 137-142

24. The R\&A, USGA (2015) The Official Rules of Golf. Available from: https://www.randa.org/en/rog/2019/pages/the-rules-of-golf

25. Laywine CF, Mullen GL (1998) Discrete mathematics using Latin squares. Wiley Interscience, New York

26. Bartlett RM, Wheat J, Robins M (2007) Is movement variability important for sports biomechanists? Sports Biomech 6:224-243

27. Kruskal JB (1964) Multidimensional scaling by optimizing goodness of fit to a nonmetric hypothesis. Psychometrika 29(1):1-27

Publisher's Note Springer Nature remains neutral with regard to jurisdictional claims in published maps and institutional affiliations. 